\title{
Analysis of Alignment of TEM Image using ECC
}

\author{
Garima Goyal \\ Assistant Professor, Department of Information Science \& Engineering Jyothy Institute of Technology, \\ Bangalore, India
}

\begin{abstract}
TEM images are rapidly gaining prominence in various sectors like life sciences, pathology, medical science, semiconductors, forensics, etc. Hence, it is important to analyse the methods which are available to improve and extend the information obtainable from TEM images of particulate samples. This paper analyses the Enhanced Correlation coefficient algorithm on nanoscopic TEM images. Here, for performance criteria, correlation coefficient is taken as a parameter. By aligning several similar images to each other so they are in register and then averaging them, an image with higher signal to noise ratio can be obtained.

Index Terms: TEM Image, Alignment, ECC, Information, Signal to Noise Ratio
\end{abstract}

\section{Introduction}

Biological samples are highly radiation sensitive, thus only low electron doses can be used to image the sample. This low dose, as well as variations in the metal stain used (if used) means images have high noise relative to the signal given by the particle being observed. It requires to align images to each other so that they are in register. As the noise is mostly randomly distributed and the underlying image features constant, by averaging the intensity of each pixel over several images only the constant features are reinforced. Typically, the optimal alignment (a translation and an in-plane rotation) to map one image onto another is calculated by crosscorrelation.

Existing alignment models are parametric [1] and their exact form highly depends on on the specific application and the strategy selected to solve the alignment problem [2], [3]. Many applications use affine transformations [4]while alternative approach can rely on homography(projective transformations)[5][6].

Image alignment aims to minimize the difference between template and original image.The usual approach to image alignment is gradient descent. A variety of other numerical algorithms such as difference decomposition [7] and linear regression [8] have also been proposed, but gradient descent is the defacto standard.

Gradient descent can be done in many ways. One is additive approach whereby additive increment to the parameters is estimated [3]. Another approach is compositional approach whereby incremental warp is estimated and is then composed with the current estimate of the warp [9].

\section{Algorithm}

ECC image alignment algorithm estimates the geometric transformation (warp) between the input and the template image and it returns the warped input image which must be close to the template one, as it is shown below. The estimated transformation is the one that maximizes the correlation coefficient between the template and the warped input image.

ECC algorithm is a direct (gradient-based) image registration algorithm. Due to gradient information, it achieves high accuracy in parameter estimation (i.e. subpixel accuracy). Its performance is invariant to global illumination changes in images since it considers the correlation coefficient (zero-mean normalized cross correlation) as an objective function.

The algorithm takes as input two unregistered images (input image, template image) and estimates the 2D geometric transformation that, applied to the input image, provides a warped image registered to the template one. The current implementation includes a pyramid-based framework thus compensating large displacements. For even larger displacements or strong geometric distortions, ECC may need an appropriate initialization. This can be done either by feature matching or through an exhaustive search scheme for a coarse alignment.

The user can enable the pyramid-based implementation as well as choose the type of transformation (translation, Euclidean, affine, and homography), the number of iteration per level and the initialization transformation (optional). 
Transform is homography

\section{Methodology \& Implementation Results}
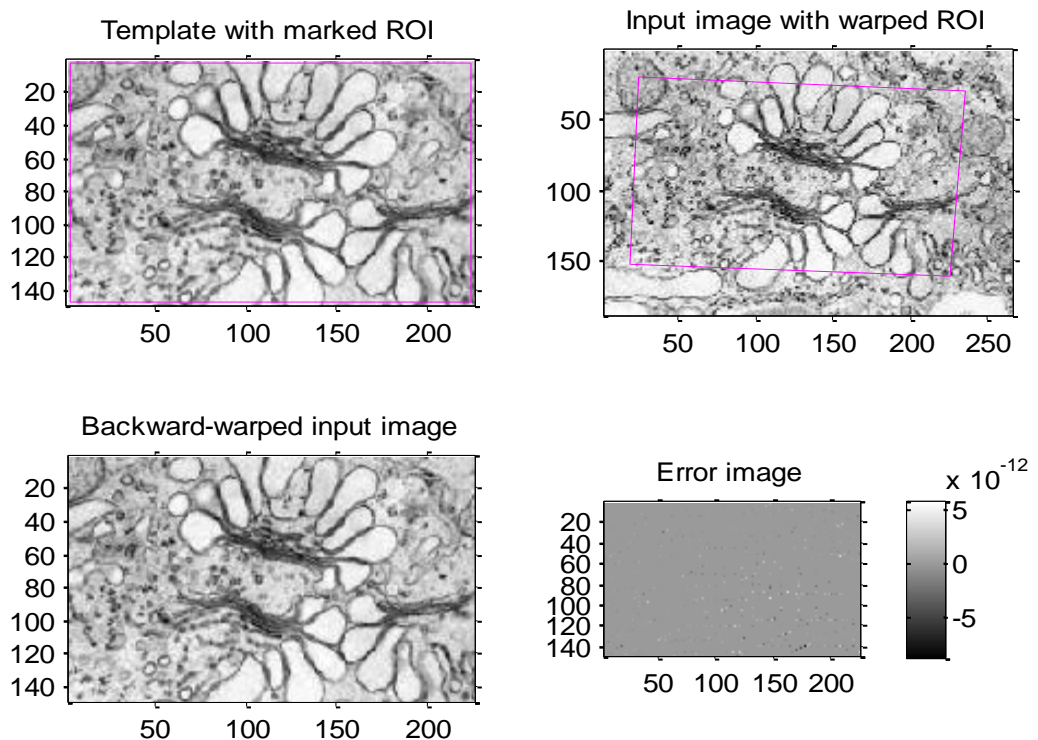

50100150200

Transform is affine
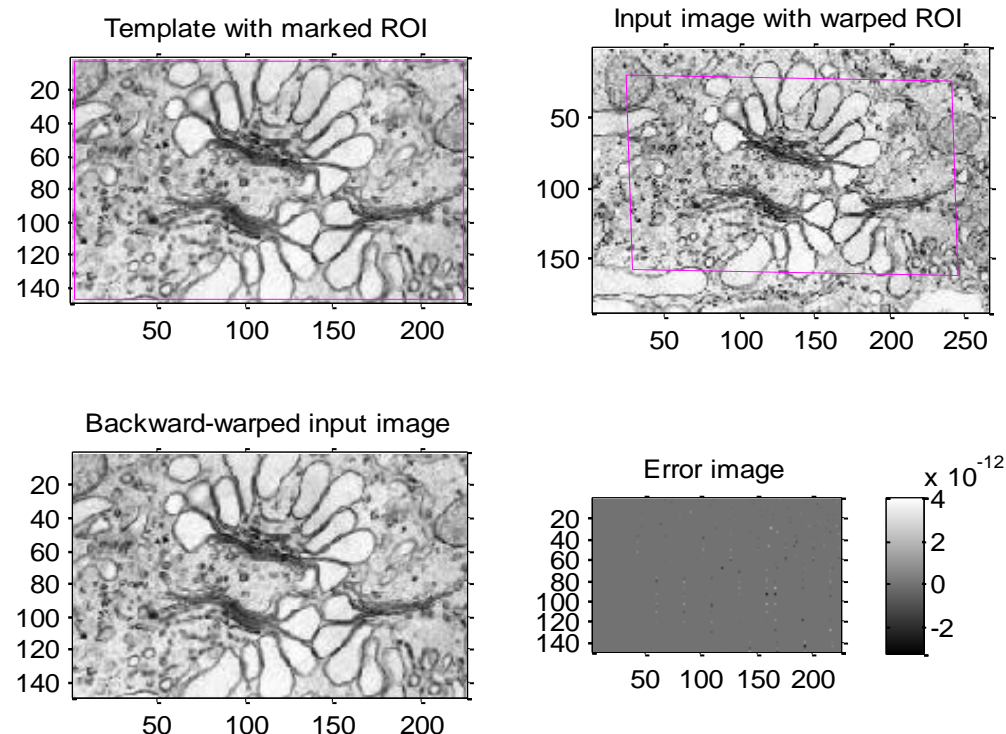

50100150200

Transform is translation
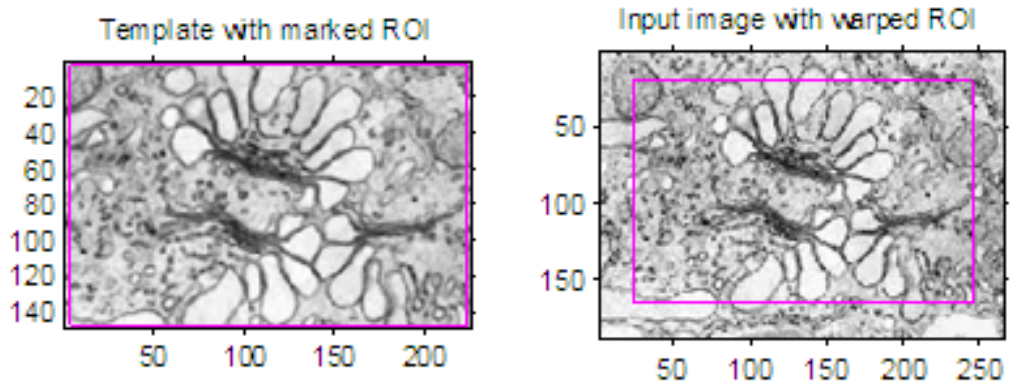

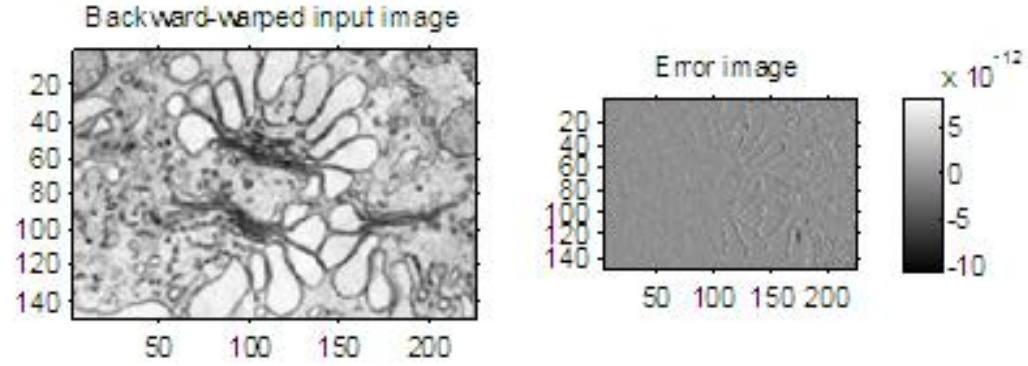

50100150200

In case of partial overlap the algorithm refines the results of feature based matching.

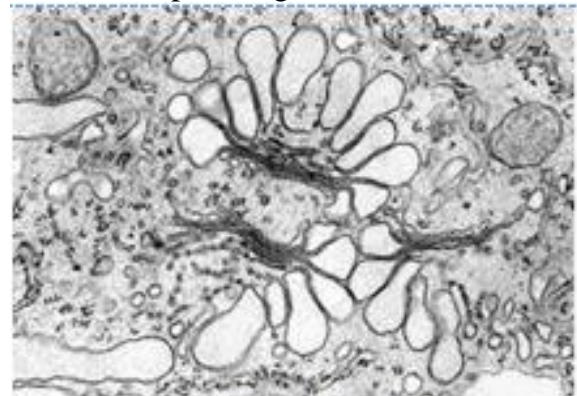

Input Image

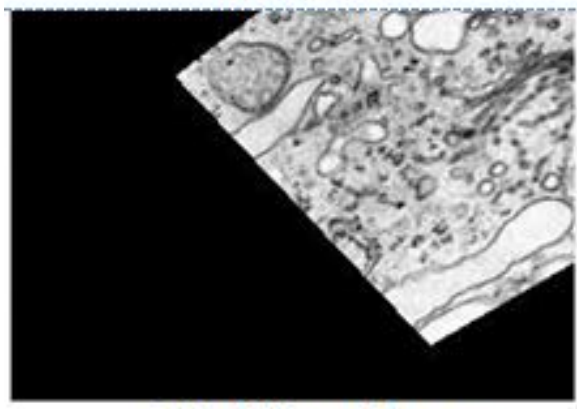

Final Warped Image

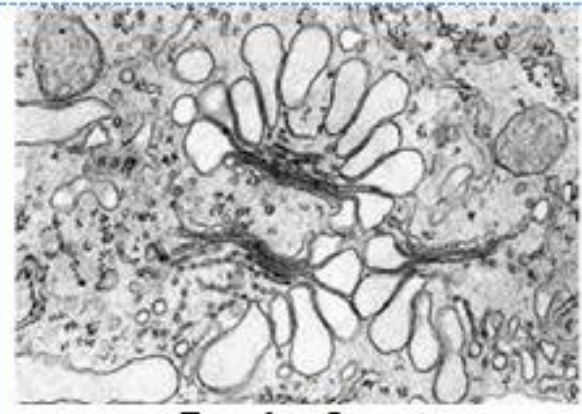

Template Image

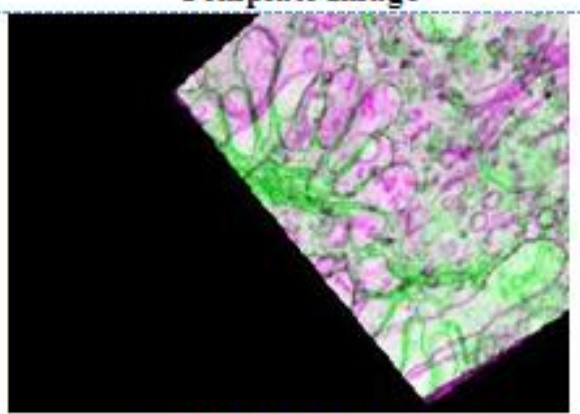

Initial Alignment

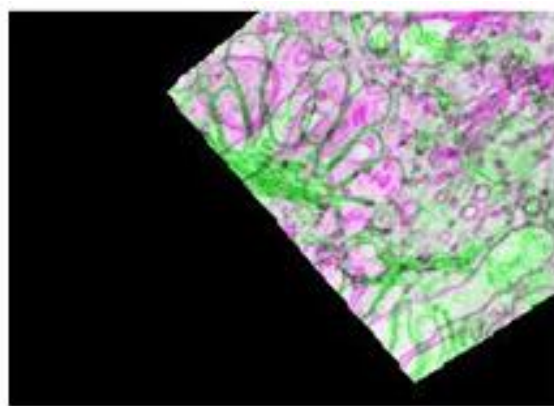

Final Alignment

\section{Conclusion}

The algorithm aimed at maximizing the enhanced correlation coefficient function which constitutes a measure that is robust against geometric and photometric distortions. The limitation of the algorithm is that it ignores a border of the template whose size is equal tofive percent of $\mathrm{M}$, where $\mathrm{M}=\operatorname{mean}$ ([size(template, 1 ), size(template,2)]).

\section{References}

[1]. R. Szeliski, H andboo k of Mathematical Models of Computer Vision, N. Paragios, Y. Chen, and O. Faugeras, eds., chapter 17. Springer, 2005.

[2]. G.D. Hager and P.N. Belhumeur, "Efficient Region Tracking with Para metric Models of Geo metry and Illumination," IEEE Trans. Pattern Analysis and Machine Intelligence, vol. 20, no. 10, pp. 1025-1039,M Oct. 1998. 
S. Baker and I. Matthews, "Lucas-Kanade 20 Years On: A Unifying Fra mework: Part 1. The Quantity Approximated, the Warp Update Rule, and the Gradient Descent Approximation,” Int'l J. Computer Vision, vol. 56, no. 3, pp. 221-255, 2004.

[3]. B.D. Lucas and T. Kanade, “An Iterative Image Registration Technique with an Application to Stereo Vision,” Proc. Seventh Int'1 Joint Conf. Artificial Intelligence, 1981.

[4]. M. Gleicher, "Projective Registration with Difference Deco mposition," Proc .IEEE Int'l Conf. Computer Vision and Pattern Recognition, 1997.

[5]. H. Shum and R. Szeliski, “Construction of Panoramic Image Mosaics with Global and Local Alignment," Int'l J. Computer Vision, vol. 36, no. 2, pp. 101130, 2000

[6]. E.Z. Ps arakis and G.D. Evangelidis, "An Enhanced Correlation-Based Method for Stereo Correspondence with Sub-Pixel Accuracy," Proc. 10th IEEE Int'l Conf. Computer Vision, 2005.

[7]. B.K.P. Horn and E.J. Weldon, "Direct Methods for Recovering Motion,’'Int'1 J. Computer Vision, vol. 2, no. 1, pp. 51-76, 1988.

16] S. Nagahdaripour and C.H. Yu, "A Generalized Brightness Change Model for Computing Optical Flow,” Proc. Fourth IEEE Int'l Conf. Computer Vision, 1993. 